\title{
Cache-miss-initiated prefetch in mobile environments
}

\author{
Hui Song, Guohong Cao* \\ Department of Computer Science and Engineering, The Pennsylvania State University, University Park, PA 16802, USA
}

Received 20 November 2003; revised 2 October 2004; accepted 12 October 2004

Available online 25 November 2004

\begin{abstract}
Prefetching has been widely used to improve system performance in mobile environments. Since prefetching consumes system resources, such as bandwidth and power, it is important to consider the system overhead when designing prefetching schemes. In this paper, we propose a cache-miss-initiated prefetch $(C M I P)$ scheme to address this issue. The CMIP scheme relies on two prefetch sets: the always-prefetch set and the miss-prefetch set. The always-prefetch set consists of the data that should always be prefetched if it is possible. The miss-prefetch set consists of the data that are closely related to the cache-missed data item. When a cache miss happens, instead of sending an uplink request to only ask for the cache-missed data item, the client requests several data items, which are within the miss-prefetch set, to reduce future cache misses. Note that the client can ask for more than one data item by an uplink request with very little additional cost. Thus, prefetching several data items in one uplink request can save additional uplink requests. We propose novel algorithms to mine the association rules and use them to construct the prefetch sets. Detailed experiments are used to evaluate the performance of the proposed scheme. Compared to the UIR scheme [G. Cao, A scalable low-latency cache invalidation strategy for mobile environments, IEEE Transactions on Knowledge and Data Engineering 15(5) (2003)] and the UIR scheme without prefetch, our CMIP scheme can greatly improve the system performance in terms of cache hit ratio, reduced uplink requests and additional traffic.
\end{abstract}

(C) 2004 Elsevier B.V. All rights reserved.

Keywords: Prefetch; Associate rule; Cache miss; Uplink; Cache management

\section{Introduction}

With the rapid growth of wireless communication technology such as IEEE 802.11 and 3G, mobile devices such as personal digital assistants (PDAs), WAP phones become more and more popular. This creates a wide range of data dissemination-based applications such as stock quotes, news, real-time weather/traffic reporting systems, etc. Many commercial companies are offering such services based on 802.11 or cellular networks. One difficulty of data dissemination is that many clients have to share a server such as the access point in 802.11 or the base station in cellular networks, which may create bottlenecks and degrade the system performance.

Data broadcasting has been shown to be an effective dissemination technique $[1,15,17,18,21]$ by utilizing

\footnotetext{
* Corresponding author.

E-mail addresses: hsong@cse.psu.edu (H. Song), gcao@cse.psu.edu (G. Cao).
}

the inherent asymmetric nature of the wireless channel, large downlink (server-to-clients) bandwidth, but less uplink (clients-to-server) bandwidth. With this technique, clients access data by simply monitoring the broadcasting channel until the requested data items appear on the channel. It has good scalability since the bandwidth consumption is independent of the number of mobile clients in the system. Although data broadcasting has many advantages, it also introduces some problems. For example, waiting for a data item to appear on the broadcast channel may increase the query latency. One way to alleviate this problem is to cache frequently accessed data on the client side. In this way, the clients can serve many requests from the local cache without sending uplink requests. This not only reduces the average data query latency but also reduces the uplink and downlink bandwidth consumption.

To further reduce the query latency, prefetching techniques can be used. In this case, when the server broadcasts data on the broadcast channel, clients can prefetch interested data without increasing the bandwidth 
consumption. Further, if the requested data item is not prefetched earlier, the client has to send an uplink request to ask for the data item when a query comes. This not only increases the query latency but also increases the uplink bandwidth requirement. Since the uplink bandwidth is very expensive in mobile environments, prefetching should be frequently used. However, prefetching consumes a lot of system resources. For example, although prefetching can make use of the broadcast channel, the clients still need to consume power to process the prefetched data. Since most mobile clients are powered by batteries, it is important to prefetch the right data when designing prefetching schemes.

In this paper, we propose a cache-miss-initiated prefetch $(C M I P)$ scheme to help the mobile clients prefetch the right data. The CMIP scheme relies on two prefetch sets: the always-prefetch set and the miss-prefetch set. The alwaysprefetch set consists of the data that should always be prefetched if possible. The miss-prefetch set consists of the data that are closely related to the cache-missed data item. When a cache miss happens, instead of sending an uplink request to only ask for the cache-missed data item, the client requests several items, which are within the miss-prefetch set, to reduce future cache misses. Note that the client can ask for more than one data item by one uplink request with little additional cost, and then prefetching several data items in one uplink request can save uplink bandwidth. We propose a novel algorithm to mine the association rules and use them to construct the prefetch sets in two steps. First, we mine the access history of the clients to obtain the association rules. Then, we use the confidence parameter of the rules to construct the prefetch sets. Detailed experiments are used to evaluate the performance of the proposed scheme. Compared to the UIR scheme [7] and the UIR scheme without prefetch, our CMIP scheme can greatly improve the system performance in terms of increased cache hit ratio, reduced uplink requests and negligible additional traffic.

The rest of the paper is organized as follows. In Section 2, we present the cache-miss-initiated prefetch (CMIP) scheme. Section 3 evaluates the performance of our CMIP scheme. Section 4 reviews previous work on prefetching and association rule mining. Section 5 concludes the paper.

\section{The cache-miss-initiated prefetch (CMIP) scheme}

Our scheme is motivated by two observations. First, a mobile client may query some data items frequently. If the requested data items are in the cache, the mobile clients can save bandwidth and power by not sending uplink requests. Thus, it is important to always prefetch these frequently accessed data. Second, data items queried during a period of time are related to each other. Hence, cache misses are not isolated events, and a cache miss is often followed by a series of cache misses. For instance, suppose data item $i_{1}$ is cache missed and data items $\left\{i_{2}, i_{3}, i_{4}\right\}$ are closely related to $i_{1}$. Fig. 1

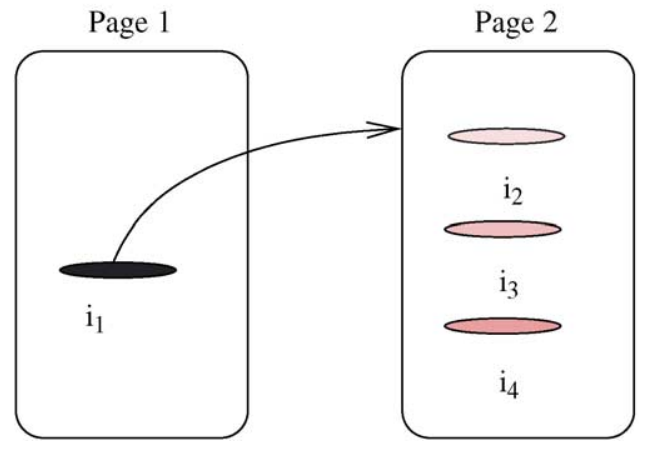

Fig. 1. The relationship between $i_{1}$ and $\left\{i_{2}, i_{3}, i_{4}\right\}$.

illustrates such a relationship between $i_{1}$ and $\left\{i_{2}, i_{3}, i_{4}\right\}$, where $i_{1}$ is a hyperlink pointing to page 2 , and $\left\{i_{2}, i_{3}, i_{4}\right\}$ are embedded objects on page 2 .

Following the cache miss of item $i_{1}$, there may be multiple cache misses because of accessing $i_{2}, i_{3}$, and $i_{4}$ sequentially. Thus, the client should prefetch items $i_{2}, i_{3}$, and $i_{4}$ after the cache miss of item $i_{1}$. After the cache miss of item $i_{1}$, the client needs to send an uplink request. Piggybacking the requests for items $i_{2}, i_{3}$, and $i_{4}$ consumes little additional uplink bandwidth, but saves three future uplink requests. Furthermore, the access latency can be greatly reduced. Based on these two observations, we propose to mine the access history of the clients, find the relationship among data items, and prefetch based on the discovered relationship.

Association rule based data mining techniques [3-5] have been proposed to find relationships among data items by analyzing a large collection of data. We propose to use these techniques to discover the association rules in the access history and use the rules to construct two prefetch sets: the always-prefetch set and the miss-prefetch set, motivated by the above two observations respectively. As the name suggests, the always-prefetch set consists of the data that should always be prefetched if possible. The missprefetch set consists of data items that will be prefetched accordingly when a cache miss happens. In the following sections, we will present our cache-miss-initiated prefetch (CMIP) scheme based on these two prefetch sets.

\subsection{Mining the access trace to obtain association rules}

In this section, we present the algorithm that generates the association rules from the access trace. Section 2.1.1 formalizes the problem, which is inspired by [3,4]. Sections 2.1.2 and 2.1.3 present the algorithm that generates association rules.

\subsubsection{The problem statement}

Suppose a client's access trace $S$ consists of a set of consecutive parts: $\left\{p_{1}, p_{2}, \ldots, p_{i}, \ldots, p_{n}\right\}$. Let $\Gamma=\left\{i_{1}, i_{2}, \ldots, i_{m}\right\}$ denote the set of data items accessed by the client. Let $S_{i}$ denote the data items contained in part $p_{i} . S_{i}$ is called 
a session and $S_{i} \subset \Gamma$. We say a session $S_{i}$ contains $X$ if $S_{i} \supseteq X$, where $X \subset \Gamma$. An association rule is defined as an implication of the form $X \Rightarrow Y$, where $X \subset \Gamma, Y \subset \Gamma$, and $X \cap Y=\varnothing X$ is called the antecedent of the rule and $Y$ is called the consequent.

A set of data items is called an itemset. The number of data items in an itemset is called the size of the itemset. An itemset of size $k$ is called a $k$-itemset. The support of an itemset $X$, support $(X)$, is defined as the percentage of sessions that contains $X$ in the client's access trace $S$. The support of an association rule $R$, support $(R)$, is defined as the support of the itemset that consists data items in both the antecedent and the consequent of the rule. For instance, the support of an association rule $R: X \Rightarrow Y$ is

$\operatorname{support}(R)=\operatorname{support}(\{X, Y\})$

The confidence of an association rule $R$, confidence $(R)$, is defined as the support of the rule divided by the support of the antecedent. For example, the confidence of the association rule $R: X \Rightarrow Y$ is

$\operatorname{confidence}(R)=\frac{\operatorname{support}(\{X, Y\})}{\operatorname{support}(X)} \times 100 \%$

Given an access trace $S$, the problem of mining association rules is to find all the association rules that have support and confidence greater than the user-specified minimum support (minsup) and minimum confidence (minconf), respectively.

The problem of discovering association rules can be decomposed into two subproblems [4]:

1. Find all the itemsets with minimum support: sup$\operatorname{port}(X) \geq$ minsup. Itemsets with minimum support are called frequent itemsets.

2. Use the frequent itemsets to generate association rules with minimum confidence.

\subsubsection{The algorithm to generate frequent itemsets}

In this section, we present the algorithm of generating frequent itemsets from the client's access trace. Table 1 shows the notation used in the algorithm.

Fig. 2 illustrates the main steps of our frequent itemsets generation algorithm. The algorithm accepts an access trace $S$ and a minimum support (minsup) as parameters. Line 1 is the first step. In this step, $S$ is analyzed to generate the frequent 1 -itemsets. This is done by calculating the support

Table 1

Notations

\begin{tabular}{ll}
\hline$k$-itemset & An itemset with $k$ items \\
$F_{k}$ & The set of frequent $k$-itemsets (those with minimum \\
& support) \\
$f_{i}, f_{j}$ & Any of the frequent $(k-1)$-itemsets within $F_{k-1}$ \\
$f_{j}$. item $m$ & Itemset $f_{j}$ 's $m$-th item \\
$c$ & A new frequent $k$-itemset got by combining a frequent \\
& $(k-1)$-itemset with one item \\
\hline
\end{tabular}

1) $F_{1} \leftarrow$ frequent 1 -itemsets $\}$

2) $k \leftarrow 2$

3) while $F_{k-1} \neq \emptyset$ do

4) $F_{k} \leftarrow \emptyset / *$ initialize $F_{k} * /$

5) for each itemset $f_{i} \in F_{k-1}$ do

6) for each itemset $f_{j} \in F_{k-1}$

7) if $f_{i}$.item $1=f_{j}$. item $_{1} \& f_{i}$. item $_{2}=f_{j}$.item $2 \& \ldots$

8) \& $\quad \& f_{i}$. item $_{k-2}=f_{j}$. item $_{k-2} \& f_{i}$. item $_{k-1}<f_{j}$. item $_{k-1}$

9) then $c \leftarrow\left\{f_{i}\right\} \cup\left\{f_{j}\right.$.item it-1 $\left._{k}\right\} ; F_{k} \leftarrow F_{K} \cup\{c\}$

10) for each (k-1)-subsets $s \in c$ do

11) if $s \notin F_{k-1}$

12) then $F_{k} \leftarrow F_{k}-\{c\}$; break

13)

14)

15)

16)

17)

18)

19)

20) $\mathrm{k}++$

21) end

22) return $\cup_{k} F_{k}$

Fig. 2. The algorithm to generate frequent itemsets.

of each data item and comparing it to the minimum support. Every data item that has minimum support forms one frequent 1-itemset.

The second step is to find all the frequent $2-, 3-, \ldots, k$ itemsets. This is done by using a loop shown from line 3 to line 21. Each iteration of the loop, say iteration $k$, generates frequent $k$-itemsets based on the $(k-1)$-itemsets generated in the previous iteration. The loop stops when no larger frequent itemsets can be generated. Inside the second step, lines 3-15 generate all the new candidate frequent $k$ itemsets out of the frequent $(k-1)$-itemsets. Lines 16-19 remove those candidate frequent $k$-itemsets that do not have minimum support. In line 22 , the algorithm returns all the frequent itemsets generated in that iteration.

\subsubsection{The algorithm to generate association rules}

The proposed algorithm generates association rules based on the frequent itemsets Only two simple kinds of association rules are generated for our purpose: one is of the form ' $\Rightarrow i_{j}$ ' and the other is of the form ' $i_{j} \Rightarrow Y$ ', where $i_{j} \in \Gamma$ and $Y \subset \Gamma$.

Table 2 shows the notations used in our algorithm. Fig. 3 illustrates the main idea of the algorithm. The algorithm accepts the frequent itemsets and a minimum confidence (minconf) as parameters. The rules are generated in two

Table 2

Notations

\begin{tabular}{ll}
\hline$R$ & The set of association rules \\
$I$ & The set of items that could be the antecedent of a rule \\
$F_{k}$ & The set of frequent $k$-itemsets \\
$N$ & The largest size of the frequent itemsets \\
$f_{j}$ & Any of the frequent $k$-itemsets within $F_{k}$ \\
$f_{j}$. item $_{i}$ & Itemset $f_{j}$ 's $i$-th item \\
\hline
\end{tabular}


1) $R \leftarrow \emptyset ; I \leftarrow \emptyset$

2) for each itemset $f_{j} \in F_{1}$

3) $I \leftarrow I \cup\left\{f_{j}\right.$.item 1$\}$

4) if support $\left(f_{j}\right.$. item $\left._{1}\right) \geq \operatorname{minconf}$

5) then $R \leftarrow R \cup\left\{" \Longrightarrow f_{j}\right.$.item $\left.1 "\right\}$

6) end

7) $k \leftarrow n$

8) while $F_{k} \neq \emptyset$ and $k>1$ do

9) for each itemset $f_{j} \in F_{k}$ do

10) for each item $f_{j}$.item $i \in f_{j}$

11) if $f_{j}$. item $_{i} \in I$ and $\frac{\operatorname{support}\left(f_{j}\right)}{\operatorname{support}\left(f_{j} \text {. } \text { item }_{i}\right)} \geq \operatorname{minconf}$

12) then $R \leftarrow R \cup\left\{f_{j}\right.$. item it $_{2} \Longrightarrow\left\{f_{j}-\left\{f_{j}\right.\right.$. item $\left.\left._{i}\right\}\right\}$ " $\}$

13) $I \leftarrow I-\left\{f_{j}\right.$. item $\left._{i}\right\}$

14) end

15) end

16) $\mathrm{k}$ - -

17) end

18) return $R$

Fig. 3. The algorithm to generate the association rules.

steps. Lines 1-6 are the first step. This step generates the first kind of rules, which are of the form ' $\Rightarrow f_{j}$.item ${ }_{1}$ ', where $f_{j}$. item $_{1} \in f_{j}$ and $f_{j} \in F_{1}$. These rules are generated from the frequent 1 -itemsets $F_{1}$ in this way. For each 1 -itemset $f_{j}$, the support of $f_{j}$.item ${ }_{1}$, which is the only one data item contained in $f_{j}$, is compared with the minimum confidence. If the support of $f_{j}$.item ${ }_{1}$ is not less than the minimum confidence, we generate a rule of the form: ' $\Rightarrow f_{j}$.item ${ }_{1}$ '. The first step also initializes the set $I$. Set $I$ is used to optimize the process of generating rules in the second step. Set $I$ consists of data items that have potential to generate rules where they are the antecedent of the rules.

Lines 7-17 are the second step. In this step, the second kind of rules of the form ' $i_{j} \Rightarrow Y$ ', where $i_{j} \in \Gamma$ and $Y \subset \Gamma$ are generated from frequent itemsets of size larger than 1 . For each frequent itemset, the rules are generated as follows. Of all the data items within the frequent itemset, one item becomes the antecedent of the rule, and all the other items become the consequent. Thus, a frequent $k$-itemset can generate at most $k$ rules. For example, suppose $\left\{i_{1}, i_{2}, i_{3}, i_{4}\right\}$ is a frequent 4-itemset. It can generate at most four rules: $i_{1} \Rightarrow\left\{i_{2}, i_{3}, i_{4}\right\}, \quad i_{2} \Rightarrow\left\{i_{1}, i_{3}, i_{4}\right\}, \quad i_{3} \Rightarrow\left\{i_{1}, i_{2}, i_{4}\right\}$, and $i_{4} \Rightarrow\left\{i_{1}, i_{2}, i_{3}\right\}$. After the rules have been generated, their confidences are calculated to determine if they have the minimum confidence. Only the rules with at least the minimum confidence are kept in the rule set $R$. For example, for the rule $i_{1} \Rightarrow\left\{i_{2}, i_{3}, i_{4}\right\}$, we need to calculate its confidence: $\operatorname{conf}=\operatorname{support}\left(\left\{i_{1}, i_{2}, i_{3}, i_{4}\right\}\right) / \operatorname{support}\left(\left\{i_{1}\right\}\right)$. If conf $\geq$ minconf, the rule holds and it will be kept in the rule set $R$. Using this method, different frequent itemsets may generate rules with the same antecedent. For example, suppose there are two frequent itemsets: a frequent 4itemset $\left\{i_{1}, i_{2}, i_{3}, i_{4}\right\}$ and a frequent 3 -itemset $\left\{i_{1}, i_{2}, i_{3}\right\}$. Both of them can generate a rule for $i_{1}$. The rule generated by the frequent 4 -itemset is ' $i_{1} \Rightarrow\left\{i_{2}, i_{3}, i_{4}\right\}$ ' and the rule generated by the frequent 3 -itemset is ' $i_{1} \Rightarrow\left\{i_{2}, i_{3}\right\}$ '.
Among the rules with the same antecedent, the one we need should have the largest itemset in the consequent of the rule. Note that a larger frequent itemset can generate a rule with the same antecedent but a larger consequent than that generated by a smaller frequent itemset. Thus, for a data item, the rules should be generated from the largest frequent itemsets first. If a rule has already been generated for a data item from a frequent itemset, say a $k$-itemset, there is no need to generate rules for the data item in any smaller frequent itemsets, such as $(k-1)$-itemsets, $(k-2)$-itemsets and so on. As in the above example, data item $i_{1}$ has two rules and the rule ' $i_{1} \Rightarrow\left\{i_{2}, i_{3}, i_{4}\right\}$ ' has a larger itemset in the consequent than in the rule ' $i_{1} \Rightarrow\left\{i_{2}, i_{3}\right\}$ '. Since $\left\{i_{2}, i_{3}, i_{4}\right\}$ contains $\left\{i_{2}, i_{3}\right\}$, the former rule has a larger view of $i_{1}$ 's relationships with other data items than the latter one. Thus, we only need the former rule for data item $i_{1}$ and do not need to generate rules, such as ' $i_{1} \Rightarrow\left\{i_{2}, i_{3}\right\}$ ', from smaller frequent itemsets.

Based on this observation, in our algorithm, the process of rule-generating starts by analyzing the largest frequent itemsets, $F_{n}$, first, and then $F_{n-1}, F_{n-2}, \ldots$, until reaching the frequent 2-itemsets $F_{2}$. In order to prevent the algorithm from generating rules for those data items that already have a rule, set $I$ is used. Set $I$ is initialized in the first step and it is used in the following way in our algorithm. Before generating a rule for a data item, say $i_{j}$, the data item $i_{j}$ is checked to see if it is in set $I$. If $i_{j}$ is found in set $I$, a rule is generated for $i_{j}$. At the same time, $i_{j}$ is deleted from the set $I$. If $i_{j}$ is not in set $I$, no rule will be generated. By using the set $I$, only one rule that has the largest consequent is generated for each data item.

\subsection{Constructing prefetch sets}

The two prefetch sets, always-prefetch set and missprefetch set, are constructed using the association rules generated above. The always-prefetch set is constructed from the first kind of rules that are of the form ' $\Rightarrow i_{1}$ ', by collecting the data items in the consequent of these rules. Since the data items in the consequent of such rules appear in most of the sessions, it is a good idea to always keep a fresh copy of them in the cache. By doing so, we can improve the cache hit ratio and reduce the number of uplink requests.

The miss-prefetch set is constructed from the second kind of rules that are of the form ' $i_{j} \Rightarrow Y$ ', where $i_{j} \in \Gamma$ and $Y \subset \Gamma$. A miss-prefetch set for the cache-missed data item is constructed when a cache miss happens. The miss-prefetch set is constructed in this way: among all the rules, the rule whose antecedent is the cache-missed data item is located and all the data items in the consequent of the rule form a miss-prefetch set of the cache-missed data item. For example, suppose data item $i_{1}$ is cache missed and we have a rule like this: ' $i_{1} \Rightarrow\left\{i_{2}, i_{3}, i_{4}\right\}$ '. The miss-prefetch set of data item $i_{1}$ is $\left\{i_{2}, i_{3}, i_{4}\right\}$. 
Note that the process of constructing the always-prefetch set and the miss-prefetch set is very fast since we only need to collect the data item IDs from a small portion of the association rules. For example, for the miss-prefetch set, we only need to check the rule of which the antecedent is the cache-missed data item, and collect the data IDs of the consequent of such rules. As a result, the construction time is negligible.

\subsection{The cache-miss-initiated prefetch (CMIP) scheme}

Our CMIP scheme prefetches data items based on the two prefetch sets. The following is a description of our CMIP scheme. Each client maintains an always-prefetch set. If the data items within the always-prefetch set are not in the cache, the client will prefetch them when they appear on the broadcast channel. If they have been updated by the server and the copies in the cache become invalid, the client prefetches them from the channel when they become available. The always-prefetch set is small. Even though we keep all the data items in the always-prefetch set within the cache, it consumes little system resources.

When a cache miss happens, the miss-prefetch set for the cache-missed data item is constructed. In case of a cache miss, the client needs to send an uplink request to the server to ask for the cache-missed data item. Instead of requesting only the cache-missed data item, all the data items within the miss-prefetch set are requested. When the server receives the request, it broadcasts all these data items on the broadcast channel. The client will prefetch and store them in its cache. By piggybacking the request of items in the miss-prefetch set, the client can save future uplink requests and reduce the access latency.

The association rules, on which the two prefetch sets are based, are highly related to the client's access pattern and the client's access pattern may change from time to time. For example, at one time, the client is interested in stock quotes. After some time, the user may like to browse the news. When the client's access pattern changes, the relationships among data items also changes. Some new relationships may show up and some old relationships may disappear. Thus, the association rules should be re-mined and updated so that they are in accordance with the client's changing access pattern. In the CMIP scheme, we re-mine and update association rules periodically to keep them fresh. This is done by adding recent accesses to the access trace and cutting off the oldest part. After re-mining the association rules, the always-prefetch set is updated immediately and the miss-prefetch sets for oncoming cache misses are constructed using the new rules.

We want to point out that, in our CMIP scheme, the data within the always-prefetch set will never be replaced or deleted from the cache, since the client has a high probability to query them in the future. But there is one exception: when updating association rules happens, some of them may become replaceable. For example, if a data item is within the old always-prefetch set, but not in the new one, it will be marked as replaceable.

To make our CMIP scheme adaptable, we need to decrease the number of prefetches when the power becomes low, since downloading a data item from the channel consumes power. Our strategy is to reduce the missinitiated-prefetch level. In this case, we need to re-mine the association rules with a higher minimum confidence (minconf) parameter, and then the rule mining algorithm can generate fewer and smaller rules. As a result, the missprefetch set becomes smaller and we only need to prefetch fewer data items from the broadcast channel. Using this strategy, we can decrease the power consumption and only prefetch the most important data items. In this paper, we assume the mobile nodes always have enough power to prefetch the data.

\section{Performance evaluation}

\subsection{The simulation model and parameters}

To evaluate the performance of the proposed methodology, we compare the CMIP scheme with the UIR scheme [7] and the UIR scheme without prefetch (called NOPRE) under various workload and system settings Our simulation is based on a simulation trace transformed from a real clientside web trace provided by Boston University [12].

\subsubsection{The simulation trace}

The web trace provided by Boston University [12] is collected for the purpose of understanding client requests for web documents Traces of actual executions of NCSA Mosaic have been collected to reflect over half a million client requests for WWW documents in the Internet. There are three types of traces: condensed, window and structure. The condensed trace contains the sequence of object (URL) requests. Each line of a condensed trace corresponds to a single URL requested by the user; it contains the machine name, the time stamp when the request was made, the URL, the size of the documents and the object retrieval time in seconds. An example of a line from a condensed trace of a client is:

cs20 785526142920156 "http://cs-www.bu.edu/lib/pica/ bu-logo.gif" 18040.484092

We utilize the condensed trace in our simulation because the client/web-server model in the web is very similar to the client/server model in mobile environments. To fit the web trace into mobile environments, we use the following strategies to transform it:

1. Clients in the web trace are mapped to mobile clients in the mobile environment.

2. URLs requested by users in the web trace are mapped to data items requested by mobile clients in the mobile environment. 
Table 3

A sample trace

\begin{tabular}{|c|c|c|c|c|c|c|c|c|c|c|}
\hline Access time & 0 & 20 & 140 & 280 & 420 & 500 & 600 & 820 & 920 & 1000 \\
\hline Data item & 0 & 1 & 2 & 422 & 1 & 2 & 423 & 10369 & 10370 & 10371 \\
\hline Access time & 2100 & 2200 & 2300 & 2400 & 2500 & 3000 & 3900 & 4000 & 4500 & 6000 \\
\hline Data item & 0 & 1 & 2 & 422 & 1 & 2 & 1384 & 1390 & 1385 & 0 \\
\hline Access time & 6200 & 6400 & 7500 & 7600 & 7700 & 7800 & 7900 & 8000 & 8100 & 8200 \\
\hline Data Item & 1 & 2 & 0 & 1 & 2 & 422 & 1 & 2 & 1384 & 1385 \\
\hline Access time & 9300 & 9400 & 9500 & 9600 & 9700 & 9800 & 9900 & 10000 & 10800 & \\
\hline Data item & 0 & 1 & 2 & 422 & 1 & 2 & 1384 & 9800 & 8744 & \\
\hline
\end{tabular}

3. The access time of a URL in the web trace is mapped to the query time in our simulation. Let the first requested URL's access time in the web trace be zero, other requested URLs' query time will be the seconds elapsed from the first request. For example, one month's real time is mapped to $60 * 60 * 24 * 30=2,592,000$ simulation seconds.

After the transformation, the trace can be used to simulate the client requests in a mobile environment. Table 3 shows a sample trace selected from the trace transformed from the web trace using the above strategies. It contains 39 data requests. The access time is in simulation seconds and each data item ID represents a URL request.

In this paper, we assume that the request patterns in mobile environments have some similarity to that of the Web traces. Although Web pages in mobile environments typically have less number of embedded objects than content-rich Web pages designed for PC access (e.g. Web pages for mobile users typically have less embedded images, advertisements, etc.), this may make our association rule-based prefetch scheme more effective. Let us look at an example of Web browsing using a PDA. It is true that there are much less embedded objects on each of the PDAfriendly Web page. This, however, makes the correlation of the objects much higher than the Internet. In Internet, each page may have tens or even hundreds of embedded objects, such as images and URL links. The correlation of these objects are pretty low since there are so many objects in one page and the user may access only a few of them. In the case of PDA-friendly Web pages, since the number of objects on one page is very small, it is very possible that the PDA user will access most of them. Thus, our association rule mining algorithm can easily find stronger correlation among these objects, since the access requests are limited to a smaller group of objects. As a result, the proposed scheme can

Table 4

The sample trace in sessions

\begin{tabular}{ll}
\hline Session \# & Data item requests \\
\hline 1 & 012242212423103691037010371 \\
2 & 0122212138413901385 \\
3 & 012 \\
4 & 0124221213841385 \\
5 & 01242212138498008744 \\
\hline
\end{tabular}

prefetch more efficiently and precisely in mobile environments.

\subsubsection{Session identification}

Before applying the association rule algorithm, we first need to construct sessions out of the simulation trace [11] uses a timeout to divide the page accesses of each user into individual sessions. If the time between page requests exceeds a certain limit, it is assumed that the user is starting a new session.

In this paper, we determine the session boundaries using an approach similar to [11], called gap-based approach. In this approach, we assume that a new session starts when the time delay between two consecutive requests is greater than a pre-specified time threshold-gap. In order to find an appropriate gap to identify the session boundaries, we have done some study on the web trace. We notice that if the time interval between two URL requests is larger than $1000 \mathrm{~s}$, then the later requested data item has little relationship with the former one. Based on this observation, we use $1000 \mathrm{~s}$ as the gap to identify sessions in our simulation. Table 4 shows that the sample trace (in Table 3) can be divided into five sessions.

Table 5 shows the frequent itemsets generated from the sample trace by applying our frequent itemsets generating algorithm to the five sessions shown in Table 4. Here, the minsup $=60 \%$. From Table 5 we can see that the largest frequent itemset is a 5-itemset.

Table 6 shows the rules generated from the sample trace by applying our rule generating algorithm to the frequent itemsets shown in Table 5. Here, the minconf $=80 \%$. As can be seen, the frequent 2- and 3-itemsets do not generate any rules. This is because after analyzing the frequent 5- and 4itemsets, all the available rules have been generated. Thus, there is no need to generate any rules from the frequent 2 and 3-itemsets.

Table 5

The frequent itemsets

\begin{tabular}{ll}
\hline 1-itemset & $\{0\},\{1\},\{2\},\{422\},\{1384\}$ \\
2-itemset & $\{0,1\},\{0,2\},\{0,422\},\{0,1384\},\{1,2\},\{1,422\}$, \\
& $\{1,1384\},\{2,422\},\{2,1384\},\{422,1384\}$ \\
3-itemset & $\{0,1,2\},\{0,1,422\},\{0,1,1384\},\{1,2,422\},\{1,2$, \\
& $1384\},\{2,422,1384\}$ \\
4-itemset & $\{0,1,2,422\},\{0,1,2,1384\},\{1,2,422,1384\}$ \\
5-itemset & $\{0,1,2,422,1384\}$ \\
\hline
\end{tabular}


Table 6

The association rules

\begin{tabular}{ll}
\hline 1-itemset & $\Rightarrow 0(100 \%)$ \\
& $\Rightarrow 1(100 \%)$ \\
& $\Rightarrow 2(100 \%)$ \\
& $\Rightarrow 422(80 \%)$ \\
2-itemset & \\
3-itemset & $0 \Rightarrow\{1,2,422\}(80 \%)$ \\
4-itemset & $1 \Rightarrow\{0,2,422\}(80 \%)$ \\
& $2 \Rightarrow\{0,1,422\}(80 \%)$ \\
& $422 \Rightarrow\{0,1,2\}(100 \%)$ \\
5-itemset & $1384 \Rightarrow\{0,1,2,422\}(100 \%)$ \\
\hline
\end{tabular}

\subsubsection{The simulation model}

The architecture of our simulation model is shown in Fig. 4. The simulation model is similar to the one used in [7, 16]. It consists of a single server that serves multiple clients.

The Server Model. To insure cache consistency, the server broadcasts invalidation reports (IRs) every $L$ seconds. IR contains the update history of the past $w$ broadcast intervals. To reduce the query latency, a number of replicated updated invalidation reports (UIRs) [7] are inserted into each IR interval. UIR contains the data items that have been updated after the last IR has been broadcast. Other messages are served on a first-come-first-serve basis. If the server is in the middle of a transmission when an IR or UIR has to be sent, the IR/UIR broadcast is deferred till the end of the current packet transmission.

The server generates a single stream of updates separated by an exponentially distributed update inter-arrival time. All updates are randomly distributed inside all the data items and only the server can update the data items. It is assumed that the bandwidth is fully utilized for broadcasting IRs and UIRs and serving client requests. The server processing time is considered to be negligible.

The Client Model. Every client, if active, listens to the IRs and UIRs to invalidate its cache accordingly. When a new request is generated, the client listens to the next IR or UIR to decide if there is a valid copy of the requested item in the cache. If there is one, the client answers the query immediately. Otherwise, a cache miss happens and the client sends an uplink request to the server. Before sending

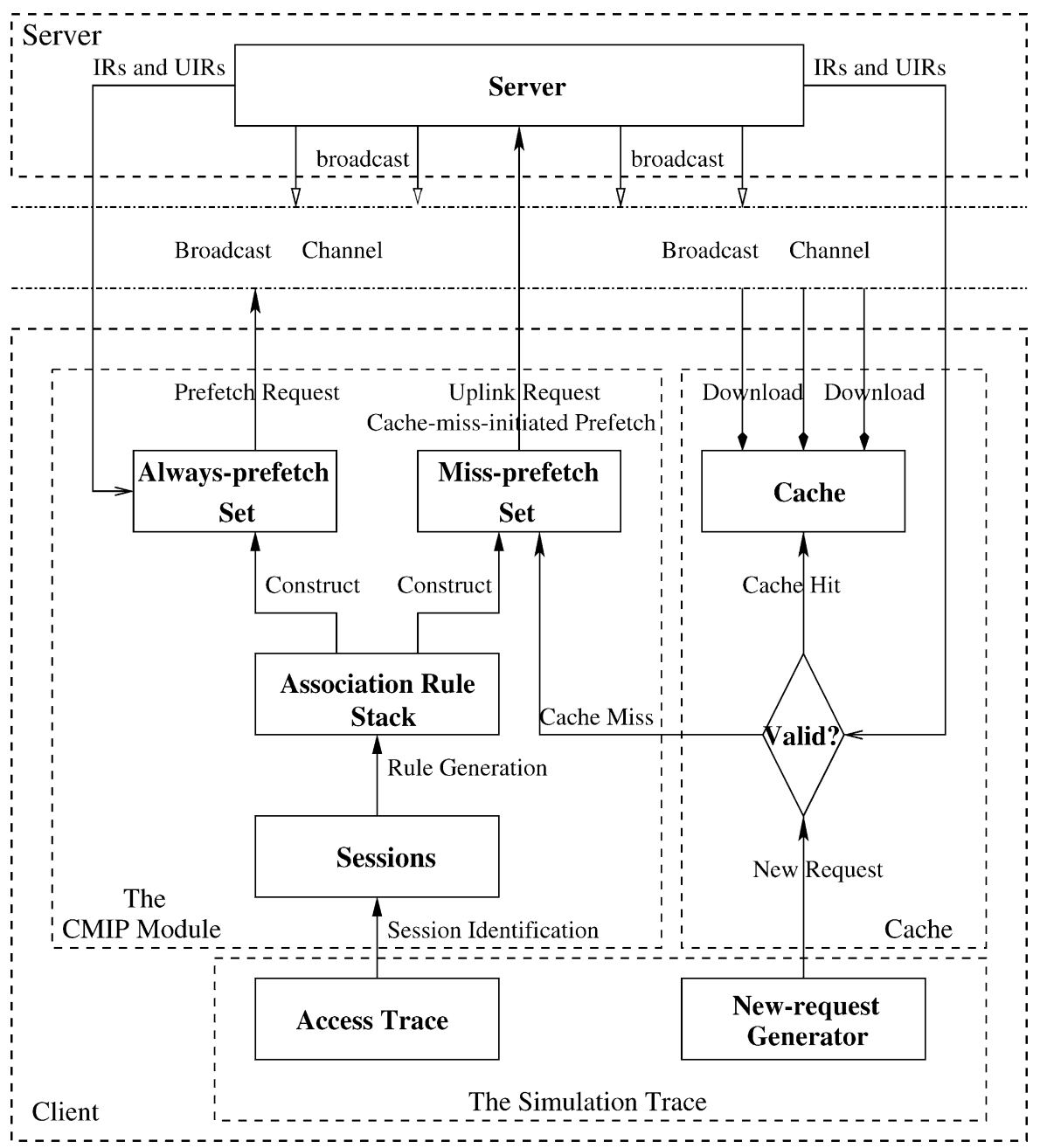

Fig. 4. The system model 
Table 7

Simulation parameters

\begin{tabular}{ll}
\hline Number of clients & 200 \\
Number of data items & 13,833 items \\
Data item size & 1024 bytes \\
Broadcast interval $(L)$ & $20 \mathrm{~s}$ \\
Broadcast bandwith & $10 \mathrm{~KB} / \mathrm{s}$ \\
Cache size & $10-300$ items \\
Broadcast window $(w)$ & 10 intervals \\
UIR replicate times $(m-1)$ & $4(5-1)$ \\
Rule minimum support $($ minsup) & $60(\%)$ \\
Rule minimum confidence & $80(\%)$ \\
(minconf) & \\
\hline
\end{tabular}

the uplink request, the requested item is sent to the CMIP module and a miss-prefetch set, corresponding to the requested item, is constructed. Then the data items in this miss-prefetch set are piggybacked to the request. After that, the uplink request is sent to the server. After receiving the uplink request, the server broadcasts the requested data on the broadcast channel. Then, the client can download them and answer the query.

The client model consists of two parts: the simulation trace and the CMIP module. The simulation trace consists of an access trace and a new-request generator. The access trace is used by the CMIP model to generate association rules and the new-request generator is used to simulate clients' requests. In the CMIP module, the access trace is divided into sessions. Then the sessions are mined using the association rule mining algorithms. The association rules with the minimum confidence (minconf) and the minimum support (minsup) are saved in the association rule stack. The always-prefetch set is constructed after obtaining the association rules. A miss-prefetch set for the cache-missed data item is constructed using the associated rules when a cache miss happens.

To keep the association rules fresh, the client updates the access trace by feeding the recent query history from the new-request generator, and re-mines the rules periodically. The always-prefetch set will be updated consequently and the new rules are used to construct miss-prefetch sets for oncoming cache misses. Most of the system parameters are listed in Table 7.

\subsection{Simulation results}

We compare the performance of our CMIP scheme with two other schemes: the UIR scheme [7] and the UIR scheme without prefetching (called NOPRE scheme). Four metrics are used to evaluate their performance: the percentage of additional traffic, the cache hit ratio, the percentage of reduced uplink requests, and the percentage of wasted prefetch.

\subsubsection{The cache hit ratio}

The performance metrics such as access latency and the uplink cost have strong relations with the cache hit ratio.
For example, if the cache hit ratio is high, the access latency can be reduced since many of the requests can be served within the cache without sending uplink requests to the server. In our simulation, we evaluate the cache hit ratio of the three schemes using various cache sizes and different number of mobile clients.

Fig. 5 compares the cache hit ratio of the CMIP scheme with those of the UIR scheme and the NOPRE scheme. As shown in Fig. 5(a), compared to the NOPRE scheme, our CMIP scheme improves the cache hit ratio greatly. This is explained as follows. The NOPRE scheme does not prefetch data items, so less data item requests can be served in the cache. When the cache is full, it uses LRU scheme to do cache replacement. However, LRU does not consider the relationships among data items. Thus important data items could be replaced by unimportant ones using the LRU scheme. For the CMIP scheme, since it has mined the relationships among data items, it knows which data items have higher future access probabilities. So it will keep these important data items in the cache longer. In this way, more client's requests can be served locally in the cache and the cache hit ratio is improved. This explains why our CMIP scheme can achieve a better performance than the NOPRE scheme in term of cache hit ratio.

Fig. 5(b) also shows that the CMIP scheme is better than the UIR scheme. This can be explains as follows. The UIR scheme is based on the cache locality: a client has a large chance to access the invalidated cache items in the near future; downloading these data items in advance should be able to increase the cache hit ratio [7]. However, the UIR scheme does not differentiate the items in the cache. Although, the UIR scheme classifies data items into hot data and cold data and treat them differently during cache management, they are treated equally in prefetching and assumed to have the same access probability in the future. But, as stated above, some of the data items within the cache are important, while others are not. The CMIP scheme differentiates the importance of the data items and keeps the important data items in the cache longer. As a result, the CMIP scheme can achieve a higher cache hit ratio than the UIR scheme.

Fig. 5(c) compares three schemes when the number of mobile clients is 200. In this case, the CMIP scheme is about $15 \%$ better than the NOPRE scheme and about $9 \%$ better than UIR in term of cache hit ratio. Although UIR scheme achieves a better cache hit ratio than NOPRE scheme, later we will see that the high cache hit ratio of the UIR scheme is at the cost of high additional traffic.

\subsubsection{The percentage of reduced uplink requests}

The percentage of reduced uplink requests is defined as the ratio of the number of saved uplink requests to the total number of requests. Since the NOPRE scheme does not prefetch, it is not available for comparison. Fig. 6 shows the percentage of reduced uplink requests using the CMIP scheme and the UIR scheme. Generally speaking, using our 

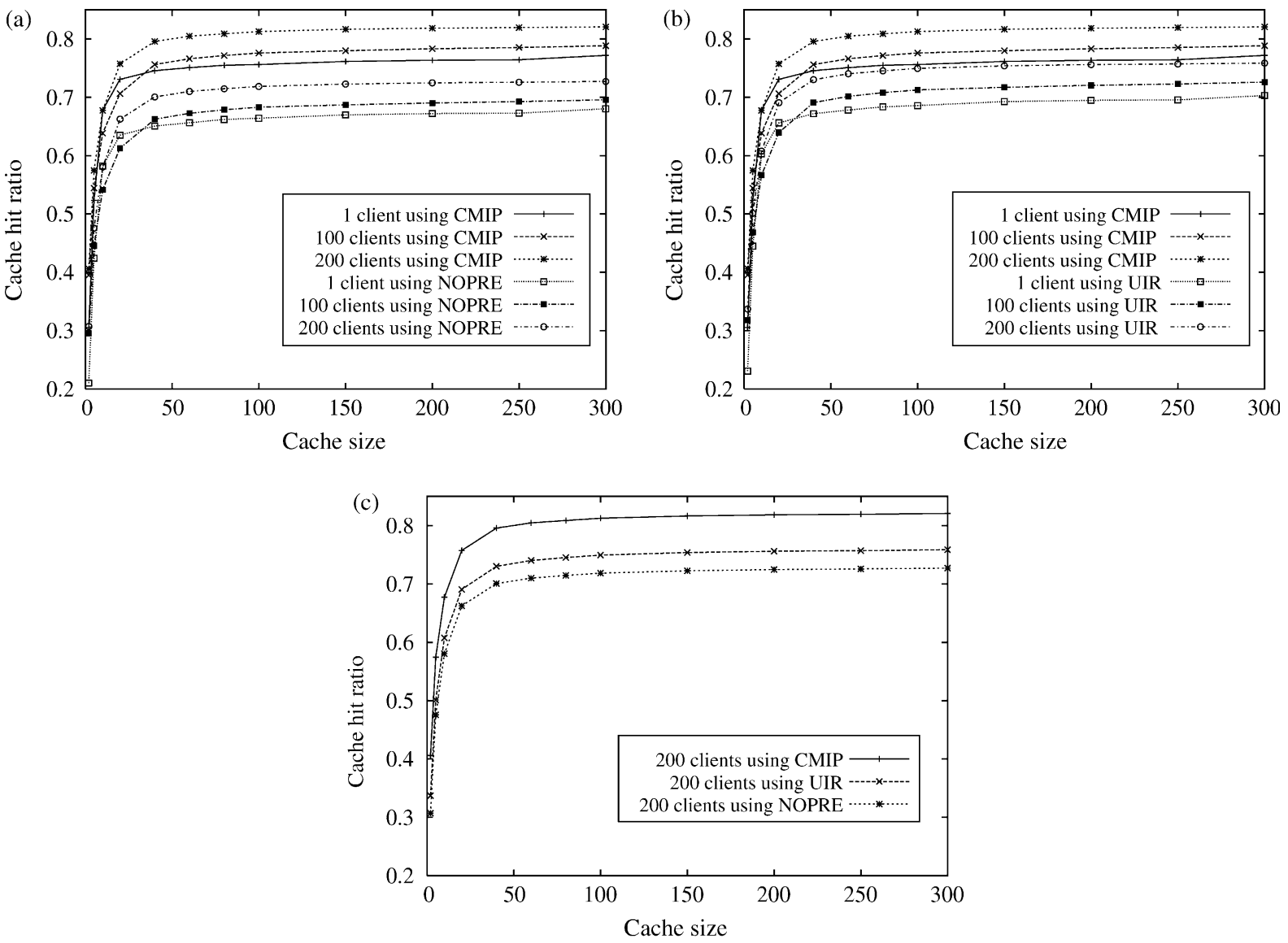

Fig. 5. The comparison of cache hit ratio using three schemes. (a) CMIP scheme vs. NOPRE scheme. (b) CMIP scheme vs. UIR scheme. (c) CMIP scheme vs. UIR scheme vs. NOPRE scheme when clients $=200$.

CMIP scheme, the percentage of reduced uplink requests increases as the cache size increases. After the cache size reaches 120, the percentage no longer changes. This can be explained as follows. As the cache size increases, more and more important data items, which have high access probability, can be stored in the cache. When the cache size is still not large enough, the cache hit ratio will increase sharply as cache size increases. As a result, the rate of reduced uplink requests is higher than the arrival rate of new requests. Thus, the percentage of reduced uplink requests has a trend of increasing in these cases. When the cache size is big enough to hold all the items within our prefetch sets, the rate of reduced uplink requests is no long significant compared to the arrival rate of new requests. Hence, the percentage of reduced uplink requests no longer changes after the cache reaches a certain size.

From Fig. 6, we also notice that although the trend of the percentage of reduced uplink requests is increasing, there are some ups and downs. For example, with 200 clients, the percentage of reduced uplink requests reaches the peak when the cache size is about 50 . As the cache size continues to increase, the percentage begins to decrease a little bit, and then increase again. This can be explained as follows. As the cache size increases, more queries can be served within the cache. So the number of reduced uplink requests keeps increasing. Depending on the access patterns of the clients, the number of reduced uplink requests may increase at a rate higher or lower than the increase of the number of requests. If the rate is higher, the percentage of reduced uplink

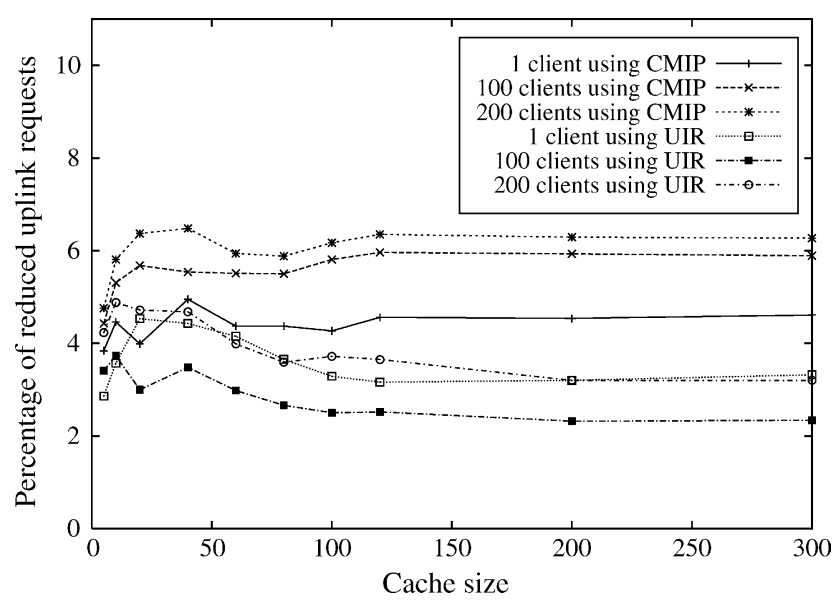

Fig. 6. The percentage of reduced uplink requests using CMIP scheme vs. UIR scheme. 
requests will increase. If the rate is lower, the percentage will decrease instead. Thus, there are some ups and downs in the percentage of reduced uplink requests, although the trend is increasing.

Fig. 6 also shows that our CMIP scheme outperforms the UIR scheme nearly all the time, no matter what the cache sizes or the number of mobile clients is. The percentage of reduced uplinks using the CMIP scheme is twice as much as that of using the UIR scheme. In term of percentage of reduced uplink request, even the worst case of the CMIP scheme is better than the best case of the UIR scheme. For instance, the CMIP scheme has the worse performance when there is only one mobile client and the percentage of reduced uplinks is about $6 \%$ (7.5\% at best). But for the UIR scheme, it achieves its best performance when there are 200 mobile clients with about $3 \%$ of reduced uplinks. This is because the CMIP scheme can better predict the future access of the clients and prefetch them in advance than the UIR scheme. Hence, the CMIP scheme can reduce the uplink requests at a percentage much higher than the UIR scheme.

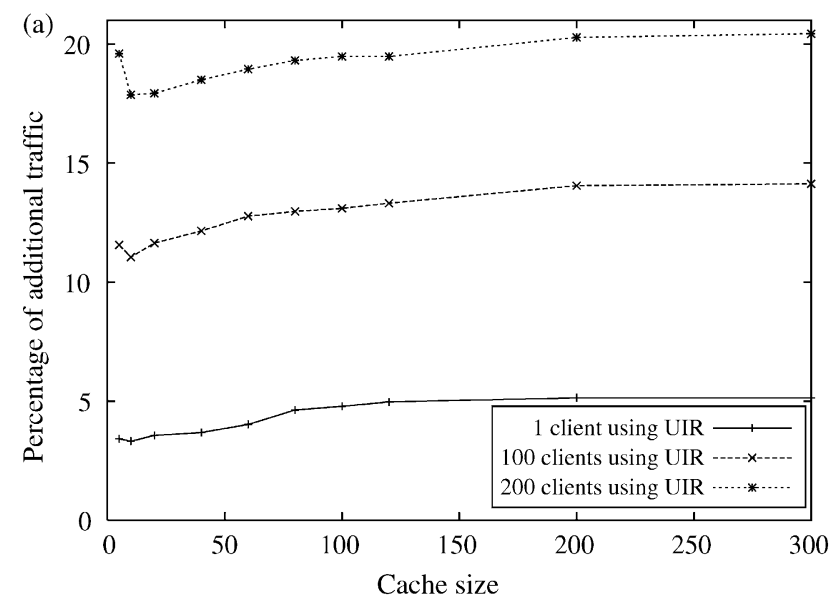

\subsubsection{The percentage of additional traffic}

The percentage of additional traffic is defined as the ratio of the number of prefetches from the broadcast channel to the number of requests. We use this metric to measure the overhead of the prefetch schemes. As we know, downloading a data item from the channel incurs overhead to the system. For example, downloading the data item consumes system resources such as bandwidth and power. So a prefetch scheme should not prefetch aggressively. Otherwise, it will consume too much resources and the overhead will offset the benefit of prefetching. This is especially important in mobile environments, where the system resources are very limited.

Note that this metric, the percentage of additional traffic, depends on the number of requests. If the number of requests is too small, the percentage will be high for an aggressive prefetch scheme that prefetches data without considering the data requesting load. This turns out to make our CMIP scheme favorable, since our scheme prefetches only when requesting data and a cache miss happens. Thus our scheme is a non-aggressive prefetch scheme and
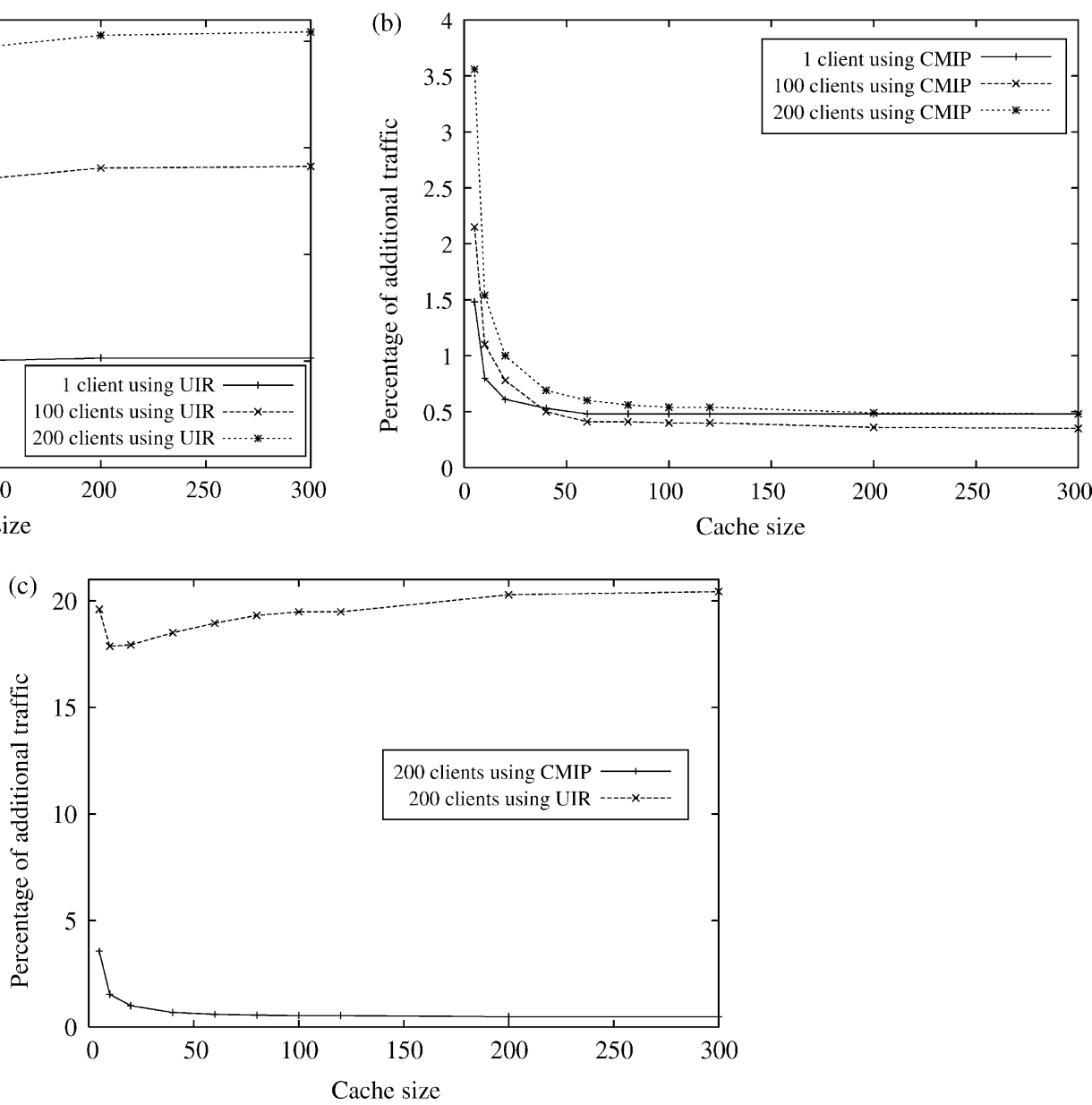

Fig. 7. The percentage of additional traffic. (a) Using UIR scheme. (b) Using CMIP scheme. (c) CMIP scheme vs. UIR scheme when the number of clients $=$ 200. 

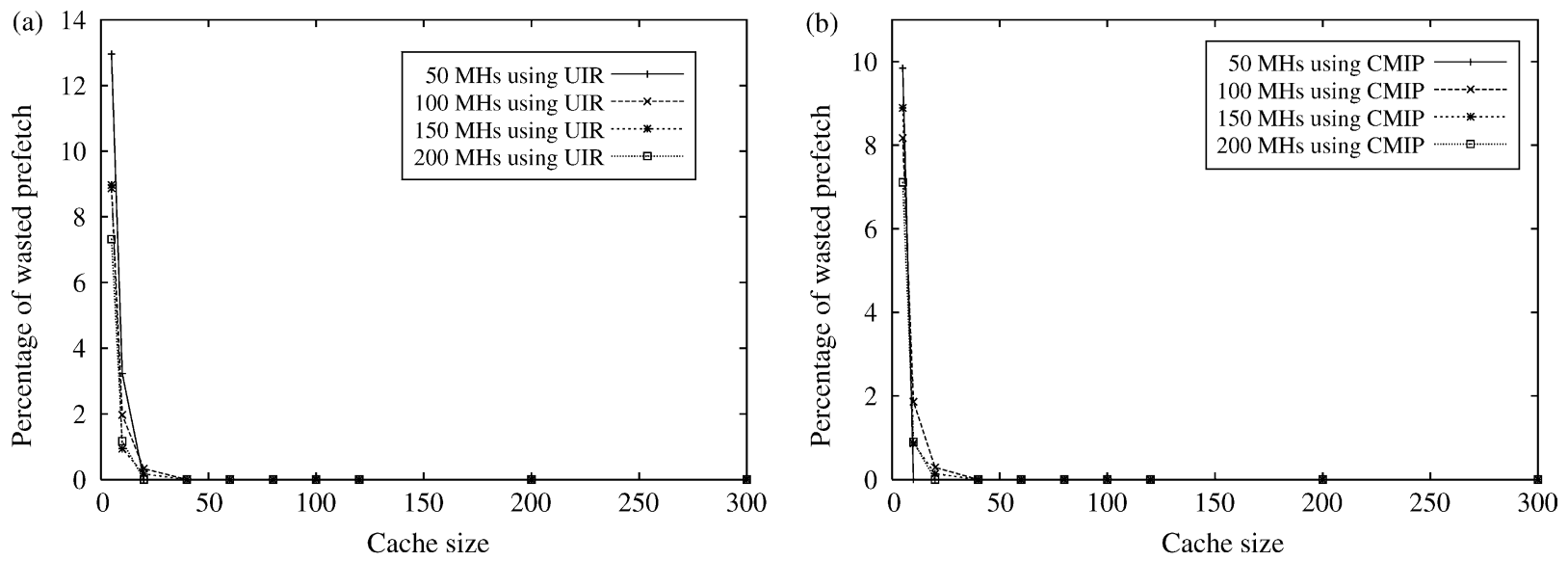

Fig. 8. The percentage of wasted prefetch. (a) Using UIR prefetch. (b) Using CMIP prefetch.

the number of prefetches is proposition to the number of requests. In addition, this metric, when combined with other metrics such as the cache hit ratio, can better describe a prefetch scheme's efficiency and prediction precision. A good prefetch scheme should be able to improve the cache hit ratio without incurring too much additional traffic (or prefetches) to the system. Fig. 7 compares the two schemes, our CMIP scheme and the UIR scheme, in term of the percentage of additional traffic.

Fig. 7(a) shows that the UIR scheme adds much additional traffic to the system. For example, the percentage of additional traffic is up to $20 \%$ when there are 200 clients. This is because the UIR scheme is an aggressive prefetch scheme. Whenever a data item within the cache has been updated and is broadcast on the channel, the client will download it and update the cache. But for the CMIP scheme, the percentage of additional traffic to the system is negligible, as shown in Fig. 7(b). For example, the percentage is lower than $0.5 \%$ when cache size becomes larger than 100 . Why the percentage of additional traffic is so small is due to the characteristic of our CMIP scheme. Using the CMIP scheme, only those data items that are within our prefetch sets are prefetched. The data items within prefetch sets are got from association rules with a high confidence and support. So the set of data items to be prefetch is small and the number of prefetches is also small. This explains why the percentage of additional traffic is negligible.

Fig. 7(c) compares the two schemes in term of the percentage of additional traffic when there are 200 mobile clients. From Fig. 7(c), we can see that our CMIP scheme incurs only a fraction of the $20 \%$ additional traffic incurred by the UIR scheme. By far, we can say that our CMIP scheme is much better than the UIR scheme and the NOPRE scheme, in terms of increased cache hit ratio, reduced uplink requests and negligible additional traffic.

\subsubsection{The percentage of wasted prefetch}

In this section, we evaluate the prefetch schemes using a metric called the percentage of wasted prefetch.
A prefetch is wasted if the prefetched data item is evacuated from the cache without being used. The percentage of wasted prefetch is defined as the ratio of the number of wasted prefetches to the total number of prefetches. This metric is used to measure the precision of the prefetch schemes. If the ratio is high, it implies that the prefetch scheme is not good and the precision of the prediction is low. On the contrary, if the ratio is low, the prefetch scheme predicts future accesses well and, in turn, can improve the system performance. Fig. 8 illustrates the percentage of wasted prefetch using two prefetch schemes (UIR and CMIP). As can be seen, the percentages of wasted prefetch for both schemes are higher when the cache size is very small. For example, when the cache size is five items, the percentage is about $13 \%$ in UIR and $10 \%$ in CMIP. This high percentage is due to the cache replacement scheme (LRU). Since the cache size is very small, it is not big enough to hold all the frequentlyaccessed data items. After a data item has been prefetched but the cache is full, a frequently-accessed data item may be removed from the cache by using LRU, which results in a high wasted prefetch percentage. On the other hand, the figure also shows that the CMIP scheme is a little better than the UIR scheme. Since the client's cache size is much larger in real world, we expect that our scheme should work well. This has also been verified by the figure. As the cache size increases, the percentage of wasted prefetch drops dramatically. When the cache size becomes larger than 50, the percentage of wasted prefetch drops to zero for both schemes. This means that almost all the prefetched data items have been used at least once before they are evacuated from the cache.

\section{Related work}

In the literature, prefetch technique is widely employed to reduce the access latency in WWW environments $[9,10$, $13,19,20]$. Ref. [20] presents a predictive prefetching 
scheme for the World Wide Web in which the server tells the clients which files are likely to be requested by the user, and the clients decide whether to prefetch these files or not based on local considerations (such as the contents of the local cache). In [19], an adaptive network prefetch scheme is proposed. This scheme predicts the files' future access probabilities based on the access history and the network condition. The scheme allows the prefetching of a file only if the access probability of the file is greater than a function of the system bandwidth, delay and retrieval time. Wcol [9] is a research prototype available on the Web. It prefetches embedded hyperlinks top-to-bottom without regard to the likelihood of use. Embedded images of prefetched pages are also prefetched. Bandwidth consumption can be controlled by configuring Wcol to prefetch no more than a certain number of hyperlinks, and no more than a certain number of images embedded within the prefetched hyperlinks. In [10], Cohen and Kaplan investigate three other types of prefetching in web: pre-resolving host-names (pre-performing DNS lookup); preconnecting (prefetching TCP connections prior to issuance of HTTP request); and pre-warming (sending a 'dummy' HTTP HEAD request to Web servers). [13] develops a new method for prefetching Web pages into the client cache. Clients send reference information to the Web server, which aggregates the reference information in near-real-time and then disperses the aggregated information to all clients, piggybacked on GET responses. The information indicates how often hyperlink URLs embedded in pages have been previously accessed relative to the embedding page. Based on the knowledge about which hyperlinks are generally popular, clients initiate the prefetching of the hyperlinks and their embedded images according to any algorithm they prefer.

Most of these work were not designed for mobile environments and did not consider the constraints of mobile environments. Recently, several prefetch schemes have been proposed as a client-side technique to reduce the access latency in mobile environments [2,8,14,22]. In [2], a simple prefetching heuristic, called PT, computes the value of a page by taking the product of the probability $(P)$ of accessing of the page with the time $(T)$ that will elapse before that page appears on the broadcast again. $P T$ finds the page in the cache with the lowest $p t$ value and replaces it with the current broadcast page if the latter has a higher $p t$ value. However, this time-based prefetch scheme is expensive to implement since it computes the $p t$ for each item in the cache at every clock tick. A similar scheme has been proposed in [14], which uses $f v$, a function of the access rate of the data item only, to evaluate the value of each data item $i$ that becomes available to the client on the channel. If there exists a data item $j$ in the client's cache such that $f v(i)>f v(j)$, data item $j$ is removed from the cache and replaced with $i$.

A prefetch scheme based on the cache locality, called UIR scheme, was proposed in [7]. It assumes that a client has a large chance to access the invalidated cache items in the near future. It proposes to prefetch these data items whenever possible to increase the cache hit ratio. In [8], Cao improves the UIR scheme by reducing some unnecessary prefetches based on the prefetch access ratio $(P A R)$. In this scheme, the client records how many times a cached data item has been accessed and prefetched, respectively. It then calculates the $P A R$, which is the number of prefetches divided by the number of accesses, for each data item. If the $P A R$ is less than one, it means that the data item has been accessed a number of times and hence the prefetching is useful. The clients can mark data items as non-prefetching when PAR $>b$, where $b$ is a system tuning factor. The scheme proposes to change the value of $b$ dynamically according to power consumption. This can make the prefetch scheme adaptable, but no clear methodology as to how and when $b$ should be changed has been discussed. Yin and Cao [22] proposed a power-aware prefetch scheme, called value-based adaptive prefetch $(V A P)$ scheme, which can dynamically adjust the number of prefetches based on the current energy level to prolong the system running time. The VAP scheme defines a value function that can optimize the prefetch cost to achieve better performance.

These existing schemes ignore some characteristics of the mobile environments: (1) a mobile client may query some data items frequently, (2) data items queried during a period of time are related to each other, (3) cache misses are not isolated events, and a cache miss is often followed by a series of cache misses, (4) when a cache miss happens, piggybacking several requests in the cache-miss-initiated uplink request consumes little additional uplink bandwidth, but reduces several future uplink requests and reduces the access latency. In this paper, we addressed these issues by a cache-miss-initiated prefetch scheme that prefetches highly related data items. In order to find the relationships among data items, we applied association rule based data mining technique. This technique has been widely studied in a market analysis context [6]. The problem of finding association rules among items has been clearly defined by Agrawal et al. in [4]. Our context, mobile environments, has different conditions and thus a direct application of existing association rule mining algorithm [3], is not applicable:

- We are interested in rules with only one data item in the antecedent and several data items in the consequent. Our motivation is to prefetch several data items that are highly related to the cache-miss data item within the cache-miss initiated uplink request. We want to generate rules where the antecedent is one data item, the cachemissed data item, and the consequent is a series of data items, which are highly related to the antecedent. If we have such rules, we can easily find the data items that should also be piggybacked in a cache-miss initiated uplink request.

- In mobile environments, the client's computation and power resources are limited. Thus, the rule-mining process should be simple and resource inexpensive. 
It should only take a short time to mine the rules. It should have low computation overhead. However, most of the association rule mining algorithms [3,4] are complex and have high computation, resource, and time requirements in order to generate association rules.

\section{Conclusions}

Client-side prefetching technique can be used to improve system performance in mobile environments. However, prefetching also consumes a large amount of system resources such as computation power and energy. Thus, it is very important to only prefetch the right data. In this paper, we proposed a cache-miss-initiated prefetch (CMIP) scheme to help the mobile clients prefetch the right data. The CMIP scheme relies on two prefetch sets: the alwaysprefetch set and the miss-prefetch set. Novel association rule based algorithms were proposed to construct these prefetch sets. When a cache miss happens, instead of sending an uplink request to ask for the cache-missed data item only, the client requests several items, which are within the miss-prefetch set, to reduce future cache misses. Detailed experimental results verified that the CMIP scheme can greatly improve the system performance in terms of increased cache hit ratio, reduced uplink requests and negligible additional traffic. Results also show that the percentage of wasted prefetch is nearly zero when the cache size is big enough.

\section{Acknowledgements}

We would like to thank the anonymous referees whose insightful comments helped us to improve the presentation of the paper. This work was supported in part by the National Science Foundation (CAREER CCR-0092770 and ITR-0219711).

\section{References}

[1] S. Acharya, S. Muthukrishnan, Scheduling on-demand broadcasts: new metrics and algorithms, ACM MobiCom'98, October 1998, pp. 43-54.

[2] S. Acharya, M. Franklin, S. Zdonik, Prefetching from a broadcast disk, Proceedings of the International Conference on Data Engineering, February 1996, pp 276-285.
[3] R. Agrawal, R. Srikant, Fast algorithms for mining association rules in: J.B. Bocca, M. Jarke, C. Zaniolo (Eds.), Proceedings of the 20th International Conference on Very Large Data Bases, VLDB, Morgan Kaufmann, Los Altos, CA, 1994, pp. 487-499.

[4] R. Agrawal, T. Imielinski, A. Swami, Mining association rules between sets of items in large databases, in: Proceedings of the ACM SIGMOD Conference on Management of Data, Washington, DC, May 1993, pp. 207-216.

[5] T.M. Anwar, H.W. Beck, S.B. Navathe, Knowledge mining by imprecise querying: a classification-based approach, in: IEEE Eighth International Conference on Data Engineering, Phoenix, Arizona, February 1992.

[6] M.J.A. Berry, G. Linoff, Data Mining Techniques: For Marketing, Sales, and Customer Support, Wiley, New York, 1997.

[7] G. Cao, A. Scalable, Low-latency cache invalidation strategy for mobile environments, IEEE Transactions on Knowledge and Data Engineering 15 (5) (2003).

[8] G. Cao, Proactive power-aware cache management for mobile computing systems, IEEE Transactions on Computers 51 (6) (2002) 608-621.

[9] K. Chinen, S. Yamaguchi, An interactive prefetching proxy server for improvement of WWW latency, in: Proceedings of INET 97, June 1997.

[10] E. Cohen, H. Kaplan, Prefetching the means for document transfer: a new approach for reducing web latency, in: Proceedings of IEEE INFOCOM, 2000, pp. 854-863.

[11] R. Cooley, B. Mobasher, J. Srivastava, Data preparation for mining world wide web browsing patterns, Knowledge and Information Systems 1 (1) (1999) 5-32.

[12] C.R. Cunha, A. Bestavros, M.E. Crovella, Characteristics of WWW client based traces. Technical Report TR-95-010, Boston University, CS Department, Boston, MA 02215, July 1995.

[13] D. Duchamp, Prefetching hyperlinks, in: USENIX Symposium on Internet Technologies and Systems (USITS'99), 1999.

[14] V. Grassi, Prefetching policies for energy saving and latency reduction in a wireless broadcast data delivery system, in: ACM MSWIM 2000, Boston MA, 2000.

[15] S. Hameed, N. Vaidya, Efficient algorithms for scheduling data broadcast, ACM/Baltzer Wireless Networks (WINET), May 1999, pp. 183-193.

[16] Q. Hu, D. Lee, Cache algorithms based on adaptive invalidation reports for mobile environments, Cluster Computing 1998; 39-48.

[17] T. Imielinski, S. Viswanathan, B. Badrinath, Data on air: organization and access, IEEE Transactions on Knowledge and Data Engineering 9 (3) (1997) 353-372.

[18] B. Li, X. Jia, J. Xu, J. Liu, Caching and prefetching for web content distribution, IEEE Computing in Science and Engineering 6 (4) (2004).

[19] Z. Jiang, L. Kleinrock, An adaptive network prefetch scheme, IEEE Journal on Selected Areas in Communications 16 (3) (1998) 1-11.

[20] V. Padmanabhan, J. Mogul, Using predictive prefetching to improve world wide web latency, Computer Communication Review 1996; 22-26.

[21] Y. Wu, G. Cao, Stretch-optimal scheduling for on-demand data broadcasts, The 10th IEEE International Conference on Computer Communications and Networks (ICCCN), October 2001.

[22] L. Yin, G. Cao, Adaptive power-aware prefetch in wireless networks, IEEE Transactions on Wireless Communication 3(5) (2004) 16481658 . 